\title{
Reduced false memory after sleep
}

\author{
Kimberly M. Fenn, ${ }^{1,7}$ David A. Gallo, ${ }^{2,3}$ Daniel Margoliash, ${ }^{2,3,4,5}$ Henry L. Roediger III, ${ }^{6}$ \\ and Howard C. Nusbaum ${ }^{2,3,4}$
}

\begin{abstract}
${ }^{1}$ Department of Psychology, Michigan State University, East Lansing, Michigan 48824, USA; ${ }^{2}$ Department of Psychology, University of Chicago, Chicago, Illinois 60637, USA; ${ }^{3}$ Center for Cognitive and Social Neuroscience, University of Chicago, Chicago, Illinois 60637, USA; ${ }^{4}$ Neuroscience Institute, University of Chicago, Chicago, Illinois 60637, USA; ${ }^{5}$ Department of Organismal Biology and Anatomy, University of Chicago, Chicago, Illinois 60637, USA; ${ }^{6}$ Department of Psychology, Washington University, St. Louis, Missouri 63130, USA
\end{abstract}

\begin{abstract}
Several studies have shown that sleep contributes to the successful maintenance of previously encoded information. This research has focused exclusively on memory for studied events, as opposed to false memories. Here we report three experiments showing that sleep reduces false memories in the Deese-Roediger-McDermott (DRM) memory illusion. False recognition of nonstudied words was reduced after sleep, relative to an equal retention interval of wakefulness, with no change in correct recognition of studied words. These experiments are the first to show that false memories can be reduced following sleep, and they extend the benefits of sleep to include increased accuracy of episodic memory.
\end{abstract}

[Supplemental material is available online at http://www.learnmem.org.]

Recent work indicates that sleep enhances episodic memory, potentially by facilitating memory consolidation. Sleep increases recall of paired associates (Plihal and Born 1997), reverses the effects of interference (Drosopoulos et al. 2007), and protects memory against subsequent retroactive interference (Ellenbogen et al. 2006). Despite the growing literature on sleep and episodic memory, the effect of sleep on false memory has yet to be established. We used the Deese-Roediger-McDermott (DRM) (Deese 1959; Roediger and McDermott 1995) false memory task to study this issue. The DRM paradigm provides a tractable laboratory procedure to study the creation and avoidance of false memories. Participants study lists of words (e.g., bed, rest, awake, tired, dream) that are semantically associated to a given word (sleep), but this associate (the critical item) is never studied. False recognition of critical items is quite robust, sometimes equal to or greater than that of studied items. False recognition is also difficult to strategically avoid and is accompanied with high levels of confidence (for review, see Gallo 2006). Furthermore, individual differences in DRM susceptibility have been associated with false autobiographical memories in some groups (Clancy et al. 2002), providing an important link to memory phenomena outside the laboratory.

Different predictions on how sleep might affect false memory can be made based on current research. From one perspective, sleep should increase false memory. Several studies have tested memory in the DRM paradigm after delays of $30 \mathrm{sec}$ to $2 \mathrm{mo}$ and found that false memories are less likely to decline after a delay than true memories (Payne et al. 1996; Thapar and McDermott 2001; Seamon et al. 2002). These findings suggest that true memory is more susceptible to forgetting than the general associative information that can cause false memories (Brainerd and Reyna 2005). In other words, veridical memory for item-specific details decays at a faster rate than more general information or gist memory. This pattern suggests that sleep may preserve gist-based information. This prediction is consistent with the finding that DRM false recogni-

\footnotetext{
${ }^{7}$ Corresponding author.
}

E-mail kfenn@msu.edu; fax (517) 432-2476.

Article is online at http://www.learnmem.org/cgi/doi/10.1101//m.1500808. tion is heavily dependent on the medial temporal lobes (Schacter et al. 1996; Cabeza et al. 2001), regions thought to be involved in sleep-based consolidation processes (Paller and Voss 2004; Wixted 2004). Although gist can lead to errors in the DRM task, this type of information is thought to be beneficial to various cognitive activities (Schacter et al. 1998; Brainerd and Reyna 2005) and is therefore the type of information that sleep-related consolidation processes might enhance. Furthermore, the prediction that sleep might increase false memories is also consistent with recent work on finding hidden rules in complex algorithm learning. In this study, participants were more likely to discover a hidden rule if they had slept after training than if they remained awake (Wagner et al. 2004), suggesting that the ability to generalize beyond studied information is more likely to occur after sleep. Similarly, when participants are given DRM lists to remember, they may be more likely to generalize beyond the specific items and remember the common theme of the lists after sleep.

It also could be predicted that sleep should reduce false memories. Memory for studied information is improved after sleep (Plihal and Born 1997), potentially because sleep minimizes retroactive interference and resultant forgetting (Wixted 2004). Sleep may also help to actively consolidate information that supports recollection (Drosopoulos et al. 2005) and may improve source memory (cf. Johnson et al. 1993). According to the sourcemonitoring framework, memory representations can be accompanied by explicit recollection of the details of an item's context during presentation rather than a simple sense of familiarity. For example, recognizing unusual aspects of a presentation (e.g., red print) may serve as a cue, if recollected, that a particular word had been seen previously. Sleep may strengthen perceptual details of the studied information or may strengthen memory for internal cognitive processes that occurred at the time of encoding, enabling more efficient and accurate retrieval. Although the exact mechanisms of sleep-related benefits on episodic memory are still unclear, improved recollection of studied details could enhance the ability to strategically avoid false memories at retrieval. Most theories of the DRM task posit recollection-based editing processes that can help subjects avoid false memories (Gallo 2006), analogous to source monitoring processes. Lastly, Diekelmann and 
colleagues (2008) have shown that deprivation of sleep increases false memories in the DRM task, suggesting that sleep itself might decrease false memory.

To test the effect of sleep on false recognition, we conducted three experiments using the DRM paradigm. In all experiments, participants were right-handed native English speakers who reported no history of speech, hearing, or memory disorders. Participants studied DRM word lists containing 15 words each and were pseudorandomly assigned to one of two experimental or two control conditions. Detailed participant information can be found in the Supplemental material (see Supplemental Method file). All participants completed a study and test phase. Control groups completed all tasks in a single session, either at 10:00 or 22:00. Experimental groups completed the study phase either at 10:00 (12-h wake) or 22:00 (12-h sleep) and then returned $12 \mathrm{~h}$ later for the test phase, either after a waking retention interval or after an interval that included a regular sleep phase.

The presentation of each list began with the word that was most highly associated with the critical item, and each word was presented in descending associativity from the critical item. Lists were presented with a 5-sec interval between lists. In Experiment 1 , study words were presented orally, and in Experiments 2 and 3, words were displayed visually on the computer screen. Study lists for each experiment can be found in the Supplemental material (see Supplemental Word Lists document). At the conclusion of the study phase, experimental participants were permitted to leave. Control participants listened to an unrelated recorded story for $2 \mathrm{~min}$ (to minimize rehearsal) and then completed the test.

All recognition testing was visual. Individual words were displayed on the screen and participants first judged whether or not the word had been presented in any of the lists. If the participant responded that the word was new, the trial ended. If the word was judged to be old, then participants also made a remember/know judgment (Rajaram 1993; Roediger and McDermott 1995). Participants were instructed to use "remember" if they could remember anything specific about the presentation of the word and "know" if they could not remember anything specific (for complete instructions, see the Method file in the Supplemental material). Test words were presented randomly.

In the first experiment, participants studied 16 DRM lists chosen based on their strong associative strength and propensity to elicit high rates of false recognition (Roediger et al. 2001). Words were presented orally in a male voice, over Sennheiser HD570 headphones, with a 1-sec interstimulus interval (ISI). The test contained 96 items: 48 studied words, taken from list positions 1 , 8 , and 10; the 16 critical lures; and 32 unrelated lures that were taken from eight other DRM lists that were never studied $(n=4$ from each list).

We compared performance between the two 12-h retention interval groups ( $n=46$ in each) to assess the effect of sleep on illusory memory and found that false recognition of critical lures was lower after sleep than after a comparable waking interval $\left(t_{(90)}=2.0, P<0.05\right.$; Table 1$)$. It is important to note that the reduction in false recognition after sleep was selective only for items that were not studied and was not a global reduction in propensity to say that an item had been studied; correct recognition of studied items was similar for the two groups $\left(t_{(90)}=0.93, P=\right.$ 0.35). False recognition of unrelated lures was also similar $\left(t_{(90)}=\right.$ $1.2, P=0.3)$. In addition to basic memory performance, we also looked at subjective responses, but the percentage of remember responses did not vary for studied items, critical lures, or unrelated lures $\left(t_{(90)} \leq 1, P \geq 0.31\right.$, for all comparisons).

These findings suggest that illusory recognition is lower after sleep, but they require comparison of testing that occurs at opposite times in the circadian cycle. Young adults tend to be at a more
Table 1. Proportion of items recognized as "old" and SEM (in parentheses) for all three experiments

\begin{tabular}{lcccc}
\hline Condition & Studied items & Critical lures & $\begin{array}{c}\text { Unrelated } \\
\text { lures: LWs }\end{array}$ & $\begin{array}{c}\text { Unrelated } \\
\text { lures: CLs }\end{array}$ \\
\hline & & Experiment 1 & & \\
12-h wake & $0.59(0.02)$ & $0.76(0.03)$ & $0.22(0.03)$ & - \\
12-h sleep & $0.56(0.02)$ & $\mathbf{0 . 6 9}^{*}(\mathbf{0 . 0 3})$ & $0.18(0.02)$ & - \\
AM control & $0.64(0.03)$ & $0.75(0.03)$ & $0.16(0.03)$ & - \\
PM control & $0.70(0.03)$ & $0.74(0.05)$ & $0.16(0.02)$ & - \\
& & Experiment 2 & & \\
12-h wake & $0.71(0.03)$ & $0.86(0.03)$ & $0.19(0.04)$ & - \\
12-h sleep & $0.72(0.04)$ & $\mathbf{0 . 7 4 * ( 0 . 0 3 )}$ & $0.15(0.04)$ & - \\
AM control & $0.74(0.03)$ & $0.73(0.05)$ & $0.12(0.04)$ & - \\
PM control & $0.78(0.03)$ & $0.77(0.04)$ & $0.07(0.02)$ & - \\
& & Experiment 3 & & \\
12-h wake & $0.62(0.03)$ & $0.73(0.03)$ & $0.31(0.03)$ & $0.36(0.04)$ \\
12-h sleep & $0.57(0.02)$ & $\mathbf{0 . 6 3}^{*}(\mathbf{0 . 0 3})$ & $\mathbf{0 . 2 1 * *}(\mathbf{0 . 0 2})$ & $0.34(0.04)$ \\
\hline
\end{tabular}

Boldface and starred entries indicate significantly lower values when compared with those from the 12 -h wake group. ${ }^{*} P<0.05 ;{ }^{*} P<0.01$.

optimum point in their circadian cycle in the evening and have been found to perform better on certain declarative memory tasks later in the day than in the morning (May et al. 1993). Furthermore, there is evidence that false recollection of critical items does not vary based on preferred time of day in young adults (IntonsPeterson et al. 1999). However, to ensure that our results were not due to time of testing, we compared performance in the control groups. There were no significant differences between morning and evening testing in false recognition of critical lures, correct recognition of studied lures, or false recognition of unrelated lures $\left(t_{(44)} \leq 1.6, P \geq 0.12\right.$, for all comparisons).

These results suggest that after sleep there is reduced false recognition without a corresponding reduction in veridical recognition. This dissociation between true and false memories is similar to that found with other manipulations in the DRM task that have been attributed to source monitoring processes (e.g., Schacter et al. 1999; Dodson and Schacter 2001; Gallo et al. 2006). Because this was the first demonstration of a reduction in false recognition after sleep, we set out to replicate and extend the effect.

The second experiment was similar to the first experiment, but we changed the method of presentation during study; DRM lists were presented visually instead of auditorily. Previous research has shown that auditory presentation results in greater false recognition than visual, possibly because visual presentation enhances source monitoring (Smith and Hunt 1998; Gallo et al. 2001). The details of a visual presentation appear to enhance context-based recollection strategies. If sleep enhances source monitoring, perhaps by increasing the binding of contextual information to target memory information or by shifting sensitivity to the diagnostic value of such information for recognition, then one might expect a similar or even greater effect of sleep in false recognition following visual presentation.

Participants studied 10 DRM lists that varied in associative strength. Words were presented on a computer screen for 1500 msec each. The test contained 60 items: 30 studied words (list positions 1, 8, and 10); 10 critical lures; and 20 unrelated lures that were taken from 20 different DRM lists.

Experiment 2 replicated the primary finding in Experiment 1 (Table 1). False recognition of critical lures was significantly lower after sleep than after waking $\left(t_{(30)}=2.78, P<0.01\right)$, and correct recognition of list words did not vary significantly between the delay groups $\left(t_{(30)}=0.19, P=0.85\right)$. The group that was tested after sleep $(n=16)$ also showed fewer remember responses to critical 
Table 2. Remember responses in all three experiments

\begin{tabular}{|c|c|c|c|c|}
\hline Condition & Studied items & Critical lures & $\begin{array}{l}\text { Unrelated } \\
\text { lures: LWs }\end{array}$ & $\begin{array}{l}\text { Unrelated } \\
\text { lures: CLs }\end{array}$ \\
\hline \multicolumn{5}{|c|}{ Experiment 1} \\
\hline 12-h wake & $0.37(0.02)$ & $0.47(0.03)$ & $0.07(0.01)$ & - \\
\hline 12-h sleep & $0.34(0.02)$ & $0.44(0.03)$ & $0.05(0.01)$ & - \\
\hline AM control & $0.37(0.03)$ & $0.49(0.04)$ & $0.06(0.02)$ & - \\
\hline PM control & $0.45(0.03)$ & $0.52(0.05)$ & $0.03(0.01)$ & - \\
\hline \multicolumn{5}{|c|}{ Experiment 2} \\
\hline 12-h wake & $0.52(0.04)$ & $0.61(0.06)$ & $0.11(0.04)$ & - \\
\hline 12-h sleep & $0.53(0.05)$ & $0.42 *(0.06)$ & $0.07(0.02)$ & - \\
\hline AM control & $0.53(0.04)$ & $0.51(0.07)$ & $0.04(0.01)$ & - \\
\hline PM control & $0.60(0.05)$ & $0.56(0.06)$ & $0.04(0.02)$ & - \\
\hline \multicolumn{5}{|c|}{ Experiment 3} \\
\hline 12-h wake & $0.34(0.03)$ & $0.42(0.04)$ & $0.09(0.02)$ & $0.12(0.03)$ \\
\hline 12-h sleep & $0.33(0.03)$ & $0.36(0.03)$ & $0.07(0.02)$ & $0.12(0.02)$ \\
\hline
\end{tabular}

Percent of items "remembered" and SEM (in parentheses) in each experiment. Boldface and starred entries indicate significantly lower values when compared with the 12-h wake group. ${ }^{*} P<0.05$.

lures $\left(t_{(30)}=2.41, P<0.05\right)$, than the group that was tested after a waking interval $(n=16)$, an effect not found in the first experiment (Table 2).

False recognition of unrelated lures was again similar between the two groups $\left(t_{(30)}=0.78, P=0.4\right)$. Remember responses to studied items and unrelated lures were also similar $\left(t_{(30)} \leq 0.99\right.$, $P \geq 0.33$, for both comparisons). Furthermore, there were no significant differences in performance between the control groups in critical lure false recognition, correct recognition of studied items, or false recognition of unrelated lures $\left(t_{(30)} \leq 1.2, P \geq 0.23\right.$, for all comparisons). Lastly, there were no significant differences in remember responses for any item type $\left(t_{(30)} \leq 1.2, P \geq 0.25\right.$, for all comparisons).

The first two experiments show a selective reduction of false recognition of critical lures after sleep, without a corresponding loss in correct recognition. However, if participants are better able to reject false memories after sleep via enhanced source monitoring, then we might expect to see lower false recognition of unrelated lures in addition to lower false recognition of related lures (cf. Schacter et al. 1999; Gallo et al. 2006). Although there was a slight trend for this pattern in both experiments, it did not approach significance in either one. One possibility is that floor effects contributed to the lack of difference. To test this hypothesis, we used two different types of unrelated lures in Experiment 3: list words and critical lures from unstudied DRM lists. Critical lures have higher word frequencies than list words and also have higher baseline false alarm rates (Roediger and McDermott 1995). We predicted that false recognition of these items would be higher than false recognition of list words and that, because of this increased false recognition, we might see a similar pattern between sleep and wake groups on these items as on the critical lures from studied lists. Twenty DRM lists that varied in associative strength were chosen for this experiment. The lists were divided into two sets and counterbalanced within each group of participants. The test was the same for all participants so items that were targets for half of the participants were lures for the other half. All participants were part of an experimental group, either 12-h wake or 12-h sleep. Because we found, in two separate studies, that memory performance in this task did not vary based on circadian variation, there were no control groups in this experiment. During study, words were presented visually for $1500 \mathrm{msec}$. The test contained 80 items: 30 studied words (list positions 1, 8, and 10); 10 critical lures; and 40 unrelated lures (10 critical lures and 30 list words from unstudied DRM lists).
Once again, false recognition of critical lures was lower after sleep $\left(t_{(61)}=2.2, P<0.05\right)$, without a change in correct recognition $\left(t_{(61)}=0.93, P=0.19\right.$; Table 1$)$. In this experiment, there also was evidence for lower false recognition of unrelated lures after sleep. False recognition of list words from unstudied lists was lower after sleep than after waking $\left(t_{(61)}=2.7, P<0.01\right)$. However, although we predicted that we would show reduced false recognition of critical lures (from unstudied lists) after sleep, responses were similar between the two groups $\left(t_{(61)}=0.3, P=0.7\right)$. This is likely because the test contained significantly more unstudied list words $(n=30)$ than critical lures from unstudied lists $(n=10)$, giving the unstudied list words more power and making them more likely to show the effect. Although false recognition of critical lures from unstudied lists was not lower after sleep, if we collapse across item type and examine false alarms to all items from unstudied lists (both list words and critical lures), our effect is maintained. Participants in the sleep group showed lower false recognition of unrelated items, $(0.24 \pm 0.02$, mean \pm SEM), than participants in the wake group $\left(0.32 \pm 0.03, t_{(61)}=2.03, P<0.05\right)$. However, this effect was not found in the earlier experiments and therefore remains inconclusive. Remember responses were similar to critical lures, studied items, and unrelated lures $\left(t_{(61)} \leq 1, P \geq 0.32\right.$, for all comparisons).

It could be argued that in these experiments, we did not control for base-rate false alarm rates. In this experiment, we used Pr (hits - false alarms) to correct for base-rate false alarms. We calculated Pr for hits by subtracting false alarms to list words from unstudied lists from hits, and we calculated Pr for critical lures by subtracting false alarms to critical lures from unstudied lists from critical lures from studied lists. We then performed a repeatedmeasures ANOVA with the item type (corrected hits, corrected false alarms to critical lures) as a within-subjects factor, and condition (wake, sleep) as a between-subjects factor, and found a significant interaction $\left(F_{(1,61)}=8.67, P<0.01\right)$. This interaction shows that the sleep group has both lower false alarms to critical lures and higher correct recognition for studied items, suggesting improved memory accuracy (Fig. 1). There was no main effect of condition $\left(F_{(1,61)}=0.15, P=0.69\right)$ and no main effect of item type $\left(F_{(1,61)}=0.26, P=0.6\right)$. Unfortunately, we were not able

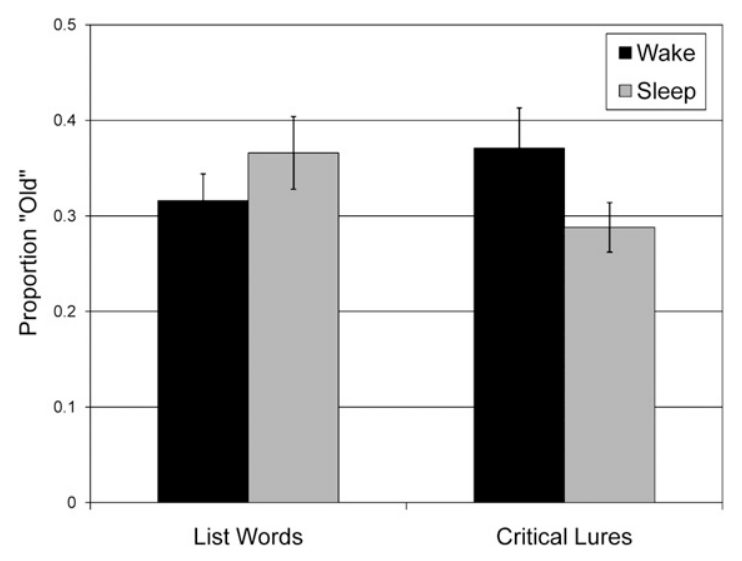

Figure 1. Data from Experiment 3, corrected for baseline false alarm rates, using $\operatorname{Pr}$ (hits - false alarms) for the sleep and wake groups. Pr for list words was calculated by subtracting false alarms to list words from unstudied lists from correct recognition of list words, and Pr for critical lures was calculated by subtracting false alarms to critical lures from unstudied lists from false alarms to critical lures from studied lists. There was a significant interaction between these factors $\left(F_{(1,61)}=8.67, P<0.01\right)$, with no main effect of either condition $\left(F_{(1,61)}=0.15, P=0.69\right)$ or item type $\left(F_{(1,61)}=0.26, P=0.6\right)$, suggesting greater memory accuracy in the sleep group. 
to perform this analysis in the earlier experiments because we did not have the appropriate control lures. Critical lures tend to have higher word frequencies and higher false alarm rates than regular list words (Roediger and McDermott 1995), so they are the only appropriate comparison group for critical lure false recognition.

These experiments provide the first evidence that false recognition is reduced after sleep. This effect cannot be explained by overall changes in response criteria or circadian variation. Young adults tend to be at a more optimal point in their circadian cycle in the evening. However, our work shows better memory performance in the morning, and our control groups suggest that this cannot be attributed to enhanced encoding in the evening. Instead, our results are consistent with the hypothesis that sleep enhanced the accuracy of source monitoring processes. Several DRM studies have found that studied words are associated with more item-specific recollections than nonstudied associates (Mather et al. 1997; Norman and Schacter 1997), a finding that gains support from neuroimaging studies (Fabiani et al. 2000; Cabeza et al. 2001). These item-specific recollections could help participants avoid false memories at retrieval. There are several possible mechanisms through which sleep might have enhanced this ability. Sleep may have actively consolidated item-specific details associated with studied items, thereby facilitating recollection-based source monitoring processes. Sleep may have strengthened sensory representations that potentially differ between true and false memory, owing to recurrent activity between the hippocampus and neocortical areas that code for sensory information (cf. Buzsaki 1998; Paller and Voss 2004). Sleep also may have provided an interval with less mental activity, during which less information was acquired. Participants who were tested after wake presumably went to class during the day and engaged in other forms of memory processing that could have increased interference on item-specific memories. Because no new information was acquired during sleep, memory accuracy may have been better simply because there was less information acquired between study and test. These different explanations for the benefits of sleep on memory have been difficult to tease apart (for review, see Wixted 2004), and our study was not designed to resolve this issue.

In contrast to the effect of sleep on false recognition, sleep did not affect true recognition of studied items. This dissociation may seem inconsistent with the idea that sleep enhanced source monitoring, but several DRM studies have shown that manipulations can suppress false recognition without affecting the ability to recognize studied items (Gallo 2006). Recognition of studied items can be influenced by both item-specific and gist-based information (Brainerd and Reyna 2005; Gallo 2006), and several studies have found that increasing perceptual details at the time of study (Schacter et al. 1999; Dodson and Schacter 2001; Gallo et al. 2006) or enhancing item-specific information (McCabe et al. 2004) can selectively suppress false memory, without affecting true memory. Moreover, these studies typically show that manipulations that enhance source monitoring reduce false recognition of both critical lures and unrelated lures, as was demonstrated in our third experiment.

In conclusion, false memories in the DRM task are very robust, and explicit manipulations of response strategies at the time of retrieval have been relatively ineffective at reducing this illusion. Nevertheless, false recognition was selectively reduced after sleep even though subjects were not given any instructions to avoid false memories. These results point toward a potentially powerful influence of sleep on episodic memory accuracy. Future work is needed to determine whether this suppression effect can generalize to autobiographical memories formed outside the laboratory. Autobiographical memories generally have more perceptual details that could potentially distinguish between true and false memories, and so the effects of sleep on autobiographical memory accuracy might be even greater than those observed here.

\section{References}

Brainerd CJ, Reyna VF. 2005. The science of false memory. Oxford University Press, New York.

Buzsaki G. 1998. Memory consolidation during sleep: A neurophysiological perspective. J Sleep Res 7: 17-23.

Cabeza R, Rao SM, Wagner AD, Mayer A, Schacter DL. 2001. Can medial temporal lobe regions distinguish true from false? An event related fMRI study of veridical and illusory recognition memory. Proc Natl Acad Sci 98: $4805-4810$.

Clancy SA, McNally RJ, Schacter DL, Lenzenweger MF, Pitman RK. 2002. Memory distortion in people reporting abduction by aliens. J Abnorm Psychol 111: 455-461.

Deese J. 1959. On the prediction of occurrence of particular verbal intrusions in immediate recall. J Exp Psychol 58: 17-22.

Diekelmann S, Landolt H-P, Lahl O, Born J, Wagner U. 2008. Sleep loss produces false memories. PLoS One 3: e3512. doi: 10.1371/journal. pone/0003512.

Dodson CS, Schacter DL. 2001. "If I had said it I would have remembered it": Reducing false memories with a distinctiveness heuristic. Psychon Bull Rev 8: 155-161.

Drosopoulos S, Wagner U, Born J. 2005. Sleep enhances explicit recollection in recognition memory. Learn Mem 12: 44-51.

Drosopoulos S, Schultze C, Fischer S, Born J. 2007. Sleep's function in the spontaneous recovery and consolidation of memories. J Exp Psychol Gen 136: $169-183$.

Ellenbogen JM, Hulbert JC, Stickgold R, Dinges DF, Thompson-Schill SL. 2006. Interfering with theories of sleep and memory: Sleep, declarative memory, and associative interference. Curr Biol 16: 1290-1294.

Fabiani M, Stadler MA, Wessels PM. 2000. True but not false memories produce a sensory signature in human lateralized brain potentials. J Cogn Neurosci 12: 941-949.

Gallo DA. 2006. Associative illusions of memory: False memory research in DRM and related tasks. Psychology Press, New York.

Gallo DA, McDermott KB, Percer JM, Roediger HL III. 2001. Modality effects in false recall and false recognition. J Exp Psychol Learn Mem Cogn 27: 339-353.

Gallo DA, Bell DM, Beier JS, Schacter DL. 2006. Two types of recollectionbased monitoring in younger and older adults: Recall-to-reject and the distinctiveness heuristic. Memory 14: 730-741.

Intons-Peterson MJ, Rocci P, West T, McLellan K, Hackney A. 1999. Age, testing at preferred or nonpreferred times (testing optimality), and false memory. J Exp Psychol Learn Mem Cogn 25: 23-40.

Johnson MK, Hashtroudi S, Lindsay DS. 1993. Source monitoring. Psychol Bull 114: 3-28.

Mather M, Henkel LA, Johnson MK. 1997. Evaluating characteristics of false memories: Remember/know judgments and memory characteristics questionnaire compared. Mem Cognit 25: 826-837.

May CP, Hasher L, Stoltzfu ER. 1993. Optimal time of day and the magnitude of age differences in memory. Psychol Sci 4: 326-330.

McCabe DP, Presamanes AG, Robertson CL, Smith AD. 2004. Item-specific processing reduces false memories. Psychon Bull Rev 11: 1074-1079.

Norman KA, Schacter DL. 1997. False recognition in younger and older adults: Exploring the characteristics of illusory memories. Mem Cognit 26: $838-848$.

Paller KA, Voss JL. 2004. Memory reactivation and consolidation during sleep. Learn Mem 11: 664-670.

Payne DG, Elie CJ, Blackwell JM, Neuschatz JS. 1996. Memory illusions: Recalling, recognizing, and recollecting events that never occurred. J Mem Lang 35: 261-285.

Plihal W, Born J. 1997. Effects of early and late nocturnal sleep on declarative and procedural memory. J Cogn Neurosci 9: 534-547.

Rajaram S. 1993. Remembering and knowing: Two means of access to the personal past. Mem Cognit 21: 89-102.

Roediger HL III, McDermott KB. 1995. Creating false memories: Remembering words not presented in lists. J Exp Psychol Learn Mem Cogn 21: 803-814.

Roediger HL III, Watson JM, McDermott KB, Gallo DA. 2001. Factors that determine false recall: A multiple regression analysis. Psychon Bull Rev 8: 385-407.

Schacter DL, Reiman E, Curran T, Yun LS, Bandy D, McDermott KB, Roediger HL III. 1996. Neuroanatomical correlates of veridical and illusory recognition memory: Evidence from positron emission tomography. Neuron 17: 267-274. 
Schacter DL, Norman KA, Koustaal W. 1998. The cognitive neuroscience of constructive memory. Annu Rev Psychol 49: 289-318.

Schacter DL, Israel L, Racine C. 1999. Suppressing false recognition in younger and older adults: The distinctiveness heuristic. J Mem Lang 40: 1-24.

Seamon JG, Luo CR, Kopecky JJ, Price CA, Rothschild L, Fung NS, Schwartz MA. 2002. Are false memories more difficult to forget than accurate memories? The effect of retention interval on recall and recognition. Mem Cognit 30: 1054-1064.

Smith RE, Hunt RR. 1998. Presentation modality affects false memory. Psychon Bull Rev 5: 710-715.
Thapar A, McDermott KB. 2001. False recall and false recognition induced by presentation of associated words: Effects of retention interval and level of processing. Mem Cognit 29: 424-432.

Wagner U, Gais S, Haider H, Verleger R, Born J. 2004. Sleep inspires insight. Nature 427: 352-355.

Wixted JT. 2004. The psychology and neuroscience of forgetting. Annu Rev Psychol 55: 235-269.

Received June 6, 2009; accepted in revised form June 23, 2009. 


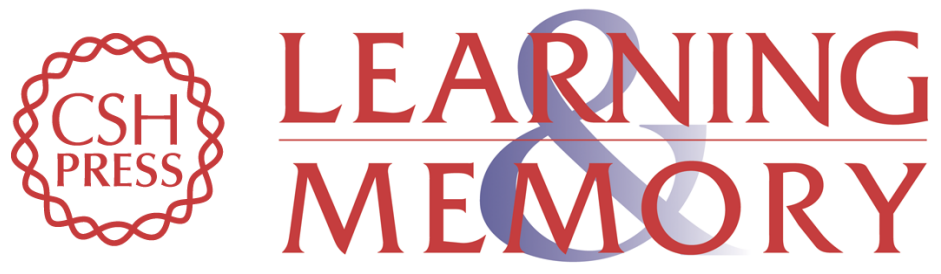

\section{Reduced false memory after sleep}

Kimberly M. Fenn, David A. Gallo, Daniel Margoliash, et al.

Learn. Mem. 2009, 16:

Access the most recent version at doi:10.1101//m.1500808

Supplemental http://learnmem.cshlp.org/content/suppl/2009/09/03/16.9.509.DC1

Material

References This article cites 32 articles, 3 of which can be accessed free at: http://learnmem.cshlp.org/content/16/9/509.full.html\#ref-list-1

License

Email Alerting Receive free email alerts when new articles cite this article - sign up in the box at the Service top right corner of the article or click here. 